\title{
Time-resolved magnetization modulation spectroscopy: A new probe of ultrafast spin dynamics
}

Citation for published version (APA):

Koopmans, B., Haverkort, J. E. M., Jonge, de, W. J. M., \& Karczewski, G. (1999). Time-resolved magnetization modulation spectroscopy: A new probe of ultrafast spin dynamics. Journal of Applied Physics, 85(9), 6763-6769. https://doi.org/10.1063/1.370191

DOI:

10.1063/1.370191

Document status and date:

Published: 01/01/1999

\section{Document Version:}

Publisher's PDF, also known as Version of Record (includes final page, issue and volume numbers)

\section{Please check the document version of this publication:}

- A submitted manuscript is the version of the article upon submission and before peer-review. There can be important differences between the submitted version and the official published version of record. People interested in the research are advised to contact the author for the final version of the publication, or visit the $\mathrm{DOI}$ to the publisher's website.

- The final author version and the galley proof are versions of the publication after peer review.

- The final published version features the final layout of the paper including the volume, issue and page numbers.

Link to publication

\section{General rights}

Copyright and moral rights for the publications made accessible in the public portal are retained by the authors and/or other copyright owners and it is a condition of accessing publications that users recognise and abide by the legal requirements associated with these rights.

- Users may download and print one copy of any publication from the public portal for the purpose of private study or research.

- You may not further distribute the material or use it for any profit-making activity or commercial gain

- You may freely distribute the URL identifying the publication in the public portal.

If the publication is distributed under the terms of Article 25fa of the Dutch Copyright Act, indicated by the "Taverne" license above, please follow below link for the End User Agreement:

www.tue.nl/taverne

Take down policy

If you believe that this document breaches copyright please contact us at:

openaccess@tue.nl

providing details and we will investigate your claim. 


\title{
Time-resolved magnetization modulation spectroscopy: A new probe of ultrafast spin dynamics
}

\author{
B. Koopmans, ${ }^{\text {a) }}$ J. E. M. Haverkort, and W. J. M. de Jonge \\ Department of Applied Physics, Eindhoven University of Technology, COBRA Research Institute, \\ P.O. Box 513, 5600 MB, Eindhoven, The Netherlands \\ G. Karczewski \\ Polish Academy of Sciences, Institute of Physics, Al. Lotników 32/46, 02-668 Warsaw, Poland
}

(Received 7 October 1998; accepted for publication 24 January 1999)

\begin{abstract}
Time-resolved magnetization modulation spectroscopy (TIMMS) is proposed as a new probe of the ultrafast spin dynamics in a wide range of magnetic media. This pump-probe technique offers a high magnetic sensitivity by imposing a high frequency modulation to the circular polarization of the pump beam. The resulting modulated magnetization is reflected in an oscillating magneto-optical signal, and is picked up using a lock-in scheme. Experimental details are discussed and a theoretical modeling of the detected signals in a TIMMS experiment is presented. We applied TIMMS to the spin dynamics of $\mathrm{CdTe} / \mathrm{CdMnTe}$ quantum well structures at room temperature. A spin scattering time of $10-15$ ps is found, almost independent of the photon energy, whereas additional time scales $(<5 \mathrm{ps})$ are found if a short period superlattice is grown in the barriers. (C) 1999 American Institute of Physics. [S0021-8979(99)01509-1]
\end{abstract}

\section{INTRODUCTION}

Experimental access to the dynamics of spins at ultrafast (subpicosecond) time scales is an enormous scientific challenge. In recent years, an increased interest in this field has been witnessed, stimulated by the development of new timeresolved magneto-optical techniques. While some of these experiments are curiosity driven, a more fundamental understanding of the fast spin dynamics is also of the utmost relevance for future progress in magnetic, magnetoelectronic and magneto-optic device technology.

The new magneto-optical pump-probe techniques that have recently been demonstrated can roughly be classified in three categories, depending on the way the pump beam perturbs the magnetic ground state: (i) "optical" or "thermal" pumping, in which a hot electron gas is excited, which heats the lattice and causes a rapid (partial) loss of the magnetization, ${ }^{1,2}$ (ii) "magnetic" pumping, in which a photoconductive switch is used to modify the magnetization by means of an ultrafast magnetic pulse,; optical" or "circularly polarized" pumping, in which spin selective optical excitations cause a change in the equilibrium spin density. ${ }^{5-7}$ All configurations have the fact that the magneto-optical Kerr or Faraday effect (or a nonlinear extension ${ }^{2}$ ) is used for probing the dynamic evolution of the magnetic state after arrival of the pump pulse in common. Related approaches are spin-polarized two-photon photoelectron spectroscopy, in which photoelectrons rather than photons are used as a probe, ${ }^{8,9}$ spin-resolved photoluminescence, ${ }^{10}$ and time-resolved semiconducting quantum interference device (SQUID) magnetometry. ${ }^{11}$

It is important to realize that the different configurations reveal different aspects of the complex spin dynamics, since

\footnotetext{
a)Electronic mail: koopmans@phys.tue.nl
}

the relaxation of a single spin depends on its orientation and energy, as well as those of all other excited electrons in the system. Exemplary in this light is the huge difference in phase space available for the relaxation of hot electrons versus that of thermal electrons. As such, further application and development of all different configurations mentioned should definitely be encouraged.

In this paper we present a new technique, time-resolved magnetization modulation spectroscopy (TIMMS), which belongs to the third class, i.e., based on a circularly polarized pump. It differs from "traditional" approaches in this class, like time-resolved Faraday spectroscopy, ${ }^{5}$ in that it applies a high frequency modulation to the circular polarization. Thus, a sensitive scheme is achieved, some of its advantages and characteristics being discussed throughout this paper. Whereas the circular pumping generally has been applied to the spin dynamics of (magnetic) semiconductors, in which the spin-orbit interaction provides a very efficient coupling of the light polarization to the spins, applications to metallic systems are hampered by a weak coupling, as a consequence of the quenched orbital momenta. We believe that the availability of a new, sensitive and complementary technique may prove itself very valuable, both in applications on the spin dynamics in (magnetic) semiconductors, and in the intriguing - though extremely complicated-magnetization dynamics in itinerant ferromagnets.

In order to asses its potential we applied TIMMS to the spin dynamics of diluted magnetic semiconductor (DMS) quantum wells (QWs). DMS materials are well recognized as spin-model systems. In a DMS, like $\mathrm{Cd}_{1-x} \mathrm{Mn}_{x} \mathrm{Te}$, a fraction of the cations is replaced by magnetic ions. While this leaves the semiconductor $(s p)$ band structure intact, it contributes local magnetic moments $(d)$ to the system. The $s p-d$ exchange interaction between these local moments and the 


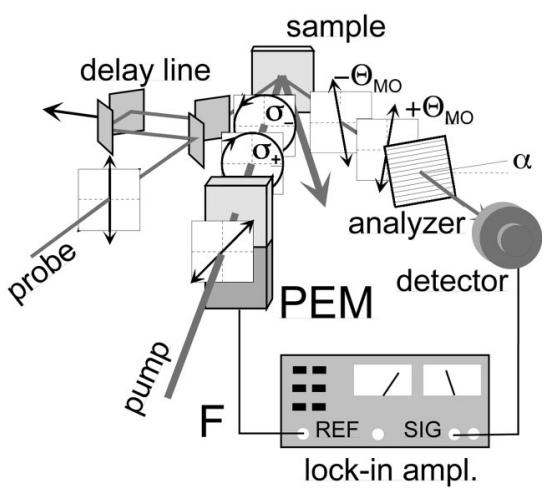

FIG. 1. Schematic diagram explaining a TIMMS experiment. A photoelastic modulator (PEM) modulates the pump between left and right-handed circular polarization $\left(\sigma_{+} \leftrightarrow \sigma_{-}\right)$. This results in a modulation of the magnetooptical rotation $\left(-\Theta_{\mathrm{MO}} \leftrightarrow+\Theta_{\mathrm{MO}}\right)$, which is picked up by a lock-in amplifier.

band electrons gives rise to a rich spectrum of collective magnetic behavior. ${ }^{12,13}$ Also, DMSs exhibit "giant" magneto-optical effects ${ }^{14}$ and a large pump-induced Faraday rotation, ${ }^{6}$ both exhibiting sharp resonances at the fundamental band gap, particularly in the case of QW structures. The recent observation of photoinduced magnetic order in III-Vbased DMS heterostructures ${ }^{15}$ illustrates the strong mutual influence of optics and magnetism in these DMS materials as well. As such, a DMS may be considered as an ideal and interesting test system for spin-dynamical investigations.

\section{METHOD}

A TIMMS setup is schematically illustrated in Fig. 1. Pump and probe pulses, at a frequency $\omega_{1}$ and $\omega_{2}$, respectively, are generated by a modelocked laser with a repetition rate $F_{\text {rep }}$. Alternatively, as was the case in our experiments, incident pulses may be split by a beamsplitter in pump and probe pulses at the same frequency $\left(\omega=\omega_{1}=\omega_{2}\right)$. The pump beam passes a photoelastic modulator (PEM) operating at a frequency of $F \ll F_{\text {rep }}$, and with its modulation axis set at $45^{\circ}$ relative to the linear polarization of the pump. Thus, the pump polarization is modulated into circular polarization with a handedness oscillating at a frequency $F$. After focusing onto the sample, a modulated magnetization/spin density is generated mediated by the spin-orbit coupling. This leads, even in the absence of a magnetic field or dc magnetization, to a magneto-optical (MO) rotation and ellipticity of the linearly polarized probe beam which is focused on the same spot on the sample. The resulting Kerr (in reflection) or Faraday (in transmission) signals oscillate at a frequency $F$ as well, and are picked up via an analyzer, detector and lock-in amplifier.

From the resemblance of the TIMMS configuration with that of a conventional Kerr or Faraday spectrometer one might intuitively expect the lock-in signals at the fundamental $\left(V_{1 F}\right)$ and second harmonic $\left(V_{2 F}\right)$ of the modulation frequency to be proportional to the induced ellipticity and rotation, respectively. The following detailed analysis shows that this is not the case!
The sample is assumed to be isotropic in the absence of the pump beam, its optical response described by a scalar $\epsilon$. The electric field amplitude of the pump $\mathbf{E}_{1}$ and probe $\mathbf{E}_{2}$ beams are written as two-dimensional (Jones) vectors:

$$
\mathbf{E}_{1}(t)=E_{1}(t)\left(\begin{array}{l}
1 / \sqrt{2} \\
1 / \sqrt{2}
\end{array}\right),
$$

and

$$
\mathbf{E}_{2}=E_{2}(t)\left(\begin{array}{l}
1 \\
0
\end{array}\right) .
$$

The two components of the Jones vector will be referred to as vertical and horizontal polarization. A common oscillating phase vector $\exp \left(\phi_{n}+i \mathbf{q}_{n} \cdot \mathbf{r}-i \omega_{n} t\right)$, with $n \in\{1,2\}$, is implicitly assumed throughout the analysis. The envelope functions $E_{n}(t)$ describe the temporal pulse shapes, which display a periodicity of $F_{\text {rep }}^{-1}, \quad E_{n}\left(t+F_{\text {rep }}^{-1}\right)=E_{n}(t)$. The transmission of the pump beam through the PEM, set to an amplitude (retardation) of $A_{0}$, is described by the Jones matrix

$$
\mathrm{M}(t)=\left(\begin{array}{cc}
1 & 0 \\
0 & \exp [i A(t)]
\end{array}\right),
$$

with $A(t)=A_{0} \cos (2 \pi F t)$. The modulation frequency $F$ has to be well below the repetition rate $F_{\text {rep }}$, so that the detected intensities can be considered as quasicontinuous. In order to show that the pump beam picks up a circular polarization modulated at $F$ while passing the PEM, the transmitted pump field, $\mathbf{E}_{1}^{\prime}(t)=\mathrm{M}(t) \cdot \mathbf{E}_{1}(t)$, is written as

$$
\mathbf{E}_{1}^{\prime}(t)=\left[A_{+}(t)\left(\begin{array}{l}
1 \\
i
\end{array}\right)+A_{-}(t)\left(\begin{array}{c}
1 \\
-i
\end{array}\right)\right] \frac{E_{1}(t)}{\sqrt{2}},
$$

with

$$
A_{ \pm}(t)=\{1 \mp i \exp [i A(t)]\} / 2 .
$$

Mediated by the spin-orbit coupling, the different absorption of the left- and right-handed circular polarization, proportional to $\left|A_{+}\right|^{2}-\left|A_{-}\right|^{2}$, causes an imbalance between spin-up $\left(N_{+}\right)$and spin-down $\left(N_{-}\right)$density of states in the interaction volume of the sample S. This in turn, induces a nonvanishing off-diagonal (nonreciprocal) element in the dielectric tensor: $i \epsilon_{x y}(t)=-i \epsilon_{y x}(t)$, which leads to a complex Faraday (or Kerr) rotation $\theta_{2}(t)$ of the transmitted (or reflected) probe beam. The relation between the pump intensity

$$
I_{1}(t)=\frac{c \sqrt{\epsilon}}{2 \pi} E_{1}^{*}(t) E_{1}(t),
$$

and the magneto-optical rotation of the probe polarization $\theta_{2}(t)$ can be described by a "response function" $\chi_{\mathrm{MO}}\left(\omega_{1}, \omega_{2}, t\right)=\chi_{\mathrm{MO}}^{\prime}+i \chi_{\mathrm{MO}}^{\prime \prime}$,

$$
\theta_{2}(t)=\int d t^{\prime} \chi_{\mathrm{MO}}\left(t-t^{\prime}\right)\left[\left|A_{+}\left(t^{\prime}\right)\right|^{2}-\left|A_{-}\left(t^{\prime}\right)\right|^{2}\right] I_{1}\left(t^{\prime}\right) .
$$

Here we dropped the frequency arguments, introduced to explicitly indicate the dependence on both frequencies. Note that $\chi_{\mathrm{MO}}$ also includes macroscopic details, such as the thickness of the crystal. 
Equation (6) is still quite general. In particular, the MO rotation may vary over the cross section of the beam, because of its intensity profile. In the following we will concentrate on average signals, introducing a beam areaaveraged laser fluence (pulse energy per area per pulse), $\mathcal{F}_{1}$

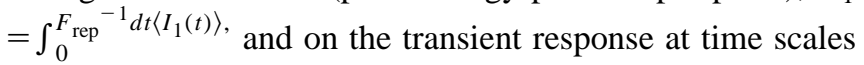
longer than the pulse width. Then, substitution of Eqs. (3) and (4) in Eq. (6), yields a transient magneto-optical rotation

$$
\theta_{2}(t, \Delta t)=\Theta_{\mathrm{MO}}(\Delta t) \sin [A(t-\Delta t)],
$$

with

$$
\Theta_{\mathrm{MO}}(\Delta t)=\mathcal{F}_{1} \chi_{\mathrm{MO}}(\Delta t) .
$$

The corresponding probe field after interaction with the sample is

$$
\mathbf{E}_{2}^{\prime}(t, \Delta t)=E_{2}(t)\left(\begin{array}{c}
1 \\
\theta_{2}(t, \Delta t)
\end{array}\right),
$$

in the limit $\Theta_{\mathrm{MO}} \ll 1$. For simplicity, we assumed the transmission of the sample to be unity. In the more general case, an additional scaling factor accounting for Fresnel factors and multiple interferences has to be included. Finally, an analyzer at angle $\alpha$ with the horizon is almost crossed with the vertical input polarization. The intensity of the probe beam after passing this analyzer reads:

$$
\begin{aligned}
I_{2}^{\prime}(t, \Delta t)= & I_{2}(t)\left\{\sin ^{2} \alpha+\sin (2 \alpha) \Theta_{\mathrm{MO}}^{\prime}(\Delta t) \sin [A(t-\Delta t)]\right. \\
& \left.+\cos ^{2} \alpha\left|\Theta_{\mathrm{MO}}(\Delta t)\right|^{2} \sin ^{2}[A(t-\Delta t)]\right\} .
\end{aligned}
$$

The corresponding Fourier components at harmonic frequencies up to $2 F$ are found by expanding $A(t-\Delta t)$ in a Taylor series in powers of $\cos (2 \pi F t)$, and assuming $\Delta t \ll F^{-1}$. This yields signals at the lock-in amplifier

$$
\begin{aligned}
& \frac{V_{\mathrm{dc}}}{V_{0}}=\sin ^{2} \alpha+\frac{1-J_{0}\left(2 A_{0}\right)}{2} \cos ^{2} \alpha\left|\Theta_{\mathrm{MO}}(\Delta t)\right|^{2}, \\
& \frac{V_{1 F}}{V_{0}}=J_{1}\left(A_{0}\right) \sin (2 \alpha) \Theta_{\mathrm{MO}}^{\prime}(\Delta t), \\
& \frac{V_{2 F}}{V_{0}}=\frac{-J_{2}\left(2 A_{0}\right)}{2}\left|\Theta_{\mathrm{MO}}(\Delta t)\right|^{2},
\end{aligned}
$$

where $J_{n}$ is the $n$th order Bessel function, and $V_{0}$ is an overall scaling factor. Now it becomes clear why almost crossing the analyzer yields an enhanced sensitivity. $I_{2}(t)$ generally contains optical noise at the lock-in frequency $1 F$ and $2 F$ due to nonidealities of the laser. Therefore, it is desirable to have a high $V_{1 F} / V_{\mathrm{dc}}$, even if this is at the cost of a lower absolute signal. This is achieved at small $\alpha\left(\left|\theta_{F}\right| \ll|\alpha|\right.$ $\ll 1)$ :

$$
\begin{aligned}
& \frac{V_{1 F}}{V_{\mathrm{dc}}} \approx 2 J_{1}\left(A_{0}\right) \frac{\Theta_{\mathrm{MO}}^{\prime}(\Delta t)}{\alpha}, \\
& \frac{V_{2 F}}{V_{\mathrm{dc}}} \approx \frac{-J_{2}\left(2 A_{0}\right)}{2}\left(\frac{|\Theta \mathrm{MO}(\Delta t)|}{\alpha}\right)^{2} .
\end{aligned}
$$

Thus, at $1 F$ an enhancement on the order of $1 / \alpha$ is realized with respect to the standard $\alpha=45^{\circ}$ configuration. At the same time the $2 F$ signal is negligible. We emphasize that
$V_{1 F}$ scales with the real part of the complex MO rotation. Information on the ellipticity is only weakly present in the experiment, via the $2 F$ signal which scales with the magnitude squared of the complex MO rotation. Only in cases where both reflection and transmission experiments can be performed, can the full complex off-diagonal tensor element be accessed. For completeness we note that the equations above are given for voltage amplitudes. Additional prefactors appear when using rms values.

Exploiting the $1 / \alpha$ enhancement factor, the optical noise can be arbitrarily decreased as long as: (i) the reduction of the absolute $1 F$ signal (scaling with $\alpha$ ) does not introduce any additional detector noise; (ii) one keeps $\alpha^{2}$ well above the extinction ratio of the analyzer $\left(10^{-5}\right.$ for good polarizers); and (iii) $\alpha \gg \Theta_{\mathrm{MO}}$ (typically $10^{-3}$ in our experiments). In practice, this means that mechanical instabilities may well become the limiting factor, particularly when using a low noise laser source.

Care should be taken to exclude spurious signals due to scattered light from the pump beam, which may occur for samples of a poor optical quality. This can easily be achieved by adjusting the analyzer parallel to the modulation axis of the beam. A completely identical analysis to the one presented above is then obtained if the polarization of the probe is set to $-\alpha$.

Starting from Eq. (6), the analysis can easily be generalized to delays short compared to the pulse width, in which case the beams have a finite temporal overlap. However, additional nonlinear optical processes may appear in this case, which will be briefly discussed in Sec. IV.

Finally, we would like to emphasize some particular advantages of our TIMMS technique. The high sensitivity and background free measurement are intrinsically related to the modulation scheme. The fact that the pump intensity is not modulated (unlike its polarization) means that nonmagnetic artifacts are excluded. Furthermore, one can take advantage of measuring the phase of the $1 F$ signal relative to that of the modulation applied by the PEM in order to estimate time scales of dynamic processes that are slow compared to the repetition rate $\left(\tau_{s} \geqslant F_{\text {rep. }}^{-1}\right)$. In that case a remainder of the signal is still present upon arrival of the next pair of pump and probe pulses, which leads to a finite signal at negative delays, its phase being a measure of the dynamic time scale. This can be seen by rewriting Eq. (7) at a small negative delay, $\Delta t=-\delta t$, for the case of a relatively long lived signal

$$
\theta_{2}(t,-\delta t)=\sum_{p=1}^{\infty} \Theta_{\mathrm{MO}}\left(p F_{\text {rep }}^{-1}\right) \sin \left[A\left(t-p F_{\text {rep }}^{-1}\right)\right] .
$$

Terms for which $\Theta_{\mathrm{MO}}\left(p F_{\text {rep }}^{-1}\right) \neq 0$ lead to a phase lag of $V_{1 F}$. The derivation of an exact expression goes beyond the scope of this paper. A simple estimate can be achieved by only considering a single term at a characteristic (relaxation) time $\tau_{s}$, defined by $\Theta_{\mathrm{MO}}\left(\tau_{s}\right) / \Theta_{\mathrm{MO}}(0)=1 / e$. Then $V_{1 F}$, which is proportional the Fourier component of $\sin \left[A\left(t-\tau_{s}\right)\right]$ to the first order in $\cos \left(2 \pi F \tau_{s}\right)$ and $\sin \left(2 \pi F \tau_{s}\right)$, is found to be

$V_{1 F} \propto \Theta_{\mathrm{MO}}^{\prime}(0) F_{\text {rep }} \tau_{s} J_{1}\left[A_{0} \cos \left(2 \pi F \tau_{s}\right)\right] \cos \left[2 \pi F t-\Phi\left(\tau_{s}\right)\right]$, 
where the phase lag $\Phi\left(\tau_{s}\right)$ is given by

$$
\tan \left[\Phi\left(\tau_{s}\right)\right]=\frac{J_{1}\left[A_{0} \sin \left(2 \pi F \tau_{s}\right)\right]}{J_{1}\left[A_{0} \cos \left(2 \pi F \tau_{s}\right)\right]} .
$$

From the property $J_{1}(0)=0$, it can easily be verified that the phase lag vanishes for $\tau_{s} \ll F^{-1}$.

\section{EXPERIMENT}

Figure 1 shows the main characteristics of our setup. A mode-locked Ti:sapphire laser tunable from 1.46 to $1.70 \mathrm{eV}$ and a repetition rate of $82 \mathrm{MHz}$ was pumped by a $5 \mathrm{~W}$ solid state laser to minimize optical noise. The latter is a big advantage since optical noise directly transfers to the signal, as discussed in the previous section. A PEM was operated at $F=50 \mathrm{kHz}$, well below the laser repetition rate as required. The PEM retardation was set to $A_{0}=2.405$, at which $J_{1}\left(A_{0}\right) \approx 0.519$. A $20 \%: 80 \%$ beamsplitter was used to split the pulses into probe and pump pulses $\left(\omega_{2}=\omega_{1}\right)$. All data are obtained at pump energies of roughly $0.3 \mathrm{~nJ}$, which corresponds to a laser fluence of $\mathcal{F}=1 \mu \mathrm{J} / \mathrm{cm}^{2}$ after focusing the pump and probe beams by a $300 \mathrm{~mm}$ lens to a spot of approximately $200 \mu \mathrm{m}$ diameter. It was verified that, except for a small thermally induced redshift of the band gap, the results did not significantly depend on the fluence in this regime. Analyzer settings between $\alpha=2^{\circ}$ and $5^{\circ}$ were chosen. All results presented were obtained in a transmission (Faraday) configuration. We checked that similar effects appear in a reflection (Kerr) geometry.

The same setup can easily be adapted to compare the spin and carrier dynamics in a pump-induced transmission (saturable adsorption) experiment. For this, a horizontally oriented polarizer (crossed with $\mathbf{E}_{1}$ ) is inserted between the PEM and the sample. This leads to an intensity modulation of the pump beam at $2 F$. The pump-induced transmission of the probe beam is then proportional to the $V_{2 F}$ signal at the lock-in amplifier.

In order to test the new TIMMS method, and because of the rich spin dynamical behavior, our first experimental studies were devoted to the spin response of DMS QWs. Various $\mathrm{CdTe} / \mathrm{Cd}_{1-x} \mathrm{Mn}_{x} \mathrm{Te}$ multiple $\mathrm{QW}$ structures were grown by molecular beam epitaxy (MBE) on $\mathrm{GaAs}(001)$ substrates, after deposition of a $1.5 \mu \mathrm{m}$ thick $\mathrm{Cd}_{0.55} \mathrm{Mg}_{0.45} \mathrm{Te}$ buffer layer, and capped by a protecting $50 \mathrm{~nm}$ thick $\mathrm{Cd}_{1-x} \mathrm{Mn}_{x}$ Te layer. For transmission experiments the GaAs, which has a fundamental band gap below that of the QW, was removed by preferential etching, and the remaining multilayer film was glued to a fused silica plate. The results of two samples, A and B, are discussed in this paper. Sample A consisted of 10 periods of a 15 monolayer (ML) CdTe well and a $30 \mathrm{ML}$ $\mathrm{Cd}_{1-x} \mathrm{Mn}_{x} \mathrm{Te}(x=0.13)$ barrier. Sample B only differed by the composition of the barrier, which was formed by a short period superlattice (SPSL); six periods of $6 \mathrm{ML} / 6 \mathrm{ML}$ $\mathrm{CdTe} / \mathrm{Cd}_{1-x} \mathrm{Mn}_{x} \mathrm{Te}$ were sandwiched between two $\mathrm{Cd}_{1-x} \mathrm{Mn}_{x}$ Te layers of $45 \mathrm{ML}($ all $x=0.13$ ).

The presence of this SPSL in the barrier strongly influences the (spin) dynamics. Due to different electron $e$ and hole $h$ masses and barrier heights, their tunneling rates are different. So, if excited over the barriers, the carriers will

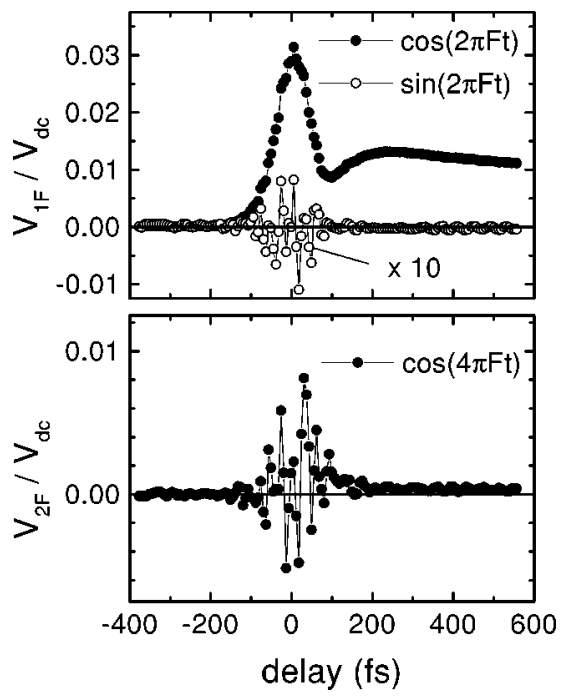

FIG. 2. Ultrafast TIMMS response at the fundamental $\left(V_{1 F}\right.$, top) and second-harmonic $\left(V_{2 F}\right.$, bottom) of the PEM modulation frequency for sample $\mathrm{B}$, at $\hbar \omega=1.625 \mathrm{eV}$, and $\alpha=3^{\circ}$. For $V_{1 F}$ both the in-phase $[\propto \cos (2 \pi F t)]$ and out-of phase $[\propto \sin (2 \pi F t)]$ signals are presented, clearly showing the wild oscillations related to the "coherent artifact.",

thermalize to the quasibound states in the SPSL, and then tunnel at a different rate to the $\mathrm{QW}$. This results in a different occupation of $e$ and $h$ states in the QW. Clearly, the additional channels add new time scales to the dynamics. By varying the parameters, this might eventually be exploited to separate $e$ and $h$ (spin) dynamics.

The fundamental optical gaps in the multilayer structure were determined by means of photoluminescence spectroscopy at $4 \mathrm{~K}$. Sample A revealed a QW exciton at $1.664 \mathrm{eV}$, and emission from the cap layer around $1.78 \mathrm{eV}$. Sample B displayed luminescence from the QW, SPSL, and cap at $1.665,1.702$ and $1.804 \mathrm{eV}$, respectively. Although many interesting phenomena in DMS materials only appear at low temperatures, and, moreover, induced Faraday signals increase by orders of magnitude at low $T$, all TIMMS investigations discussed in this paper are performed at room temperature (RT). At RT the band gaps are redshifted by approximately $0.1 \mathrm{eV}$, bringing them right into the energy window of our Ti:sapphire laser.

\section{RESULTS}

Figure 2 shows a typical example of the time-resolved TIMMS signals for our CdTe quantum structures around zero delay and at a photon energy slightly above the RT band gap of the QW. The main features of the $V_{1 F}$ signal are an almost instantaneous peak around zero delay on top of a steplike structure, which vanishes at negative delay and decays on a picosecond time scale (out of scale of this plot) at positive delay. These features are much weaker (in fact, almost absent) in the $V_{2 F}$ signal, in agreement with our theoretical analysis, Eqs. (14) and (15).

Around zero delay wild oscillations appear in the $2 F$ signal. After closer inspection, they are also present in the in-phase $1 F$ measurement [signal proportional to $\cos (2 \pi F t)$ ]. Although weaker in absolute size, they are even better ob- 

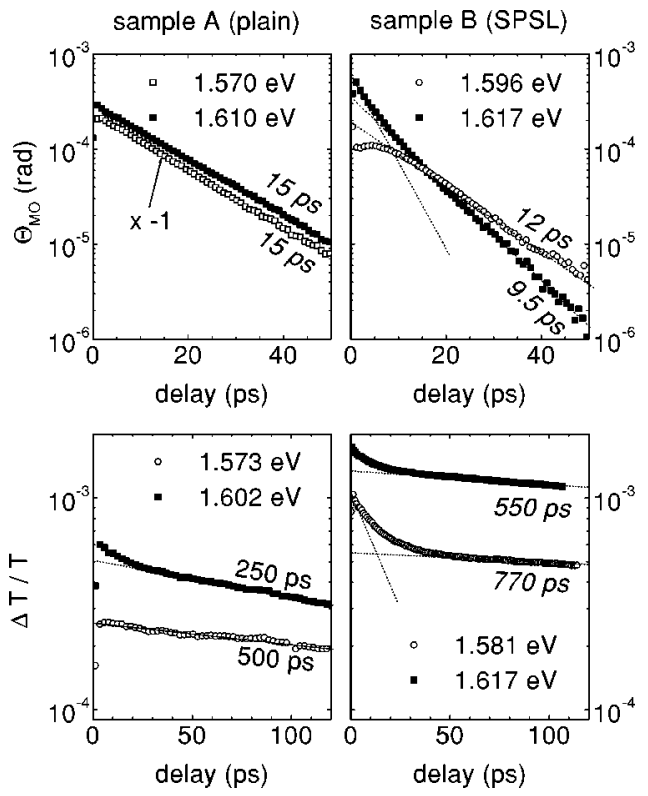

FIG. 3. Logarithmic plots of the spin and carrier dynamics in sample A (normal barriers) and B (including the SPSL barriers). The pump induced Faraday rotation (top panels) is obtained from the $V_{1 F}$ signal in a TIMMS experiment $\left(\alpha=2^{\circ}-5^{\circ}\right)$. Sample A displays a negative rotation at 1.570 $\mathrm{eV}$, the corresponding data have therefore been multiplied by -1 . The transient transmission (bottom panels) is measured at $2 F$, after inserting an additional polarizer in the pump beam. The time constants printed in italic correspond to the respective exponential decay at long delays.

served in the out-of-phase $1 F$ signal [signal proportional to $\sin (2 \pi F t)]$, due to the absence of a true anisotropy signal. In the case of transient absorption experiments this phenomenon is well known, and attributed to a so-called "coherent artifact." This parametric scattering, a third order nonlinear optical effect described by a susceptibility $\chi^{(3)}(-\omega ; \omega$, $-\omega, \omega)$, is present if the pump and probe beam have a finite temporal overlap. The oscillations are a result of the interference between the transmitted probe beam with a fraction of the pump beam, diffracted in the direction of the transmitted probe beam by a transient intensity grating. This grating is formed by the interfering pump and probe beams, which are incident on the sample at different angles. The phase of the grating is determined by the path difference between pump and probe, resulting in a full $2 \pi$ oscillation of the diffracted signal while scanning the delay length over a full wavelength, which corresponds to $\sim 4 \mathrm{fs}$ on the time axis of Fig. 2. At low sampling rates, as is the case in Fig. 2, the oscillations appear as random "noise." The width of the noise band yields an estimate of the autoconvolution width of the pump and probe pulses at the sample: $\sim 200 \mathrm{fs}$. This is doubled with respect to the laser output due to group velocity dispersion (GVD) of the optics. For experiments at a real high time resolution, some optical components should be replaced or GVD precompensation be implemented.

In order to compare the carrier and spin dynamics, and to illustrate how this dynamics is reflected in our experimental configuration, we present TIMMS as well as transient transmission data in the 1-100 ps range, both for the QW with the plain barriers (A) as for the structure including the SPSL (B). An outline of the results is displayed in Fig. 3.

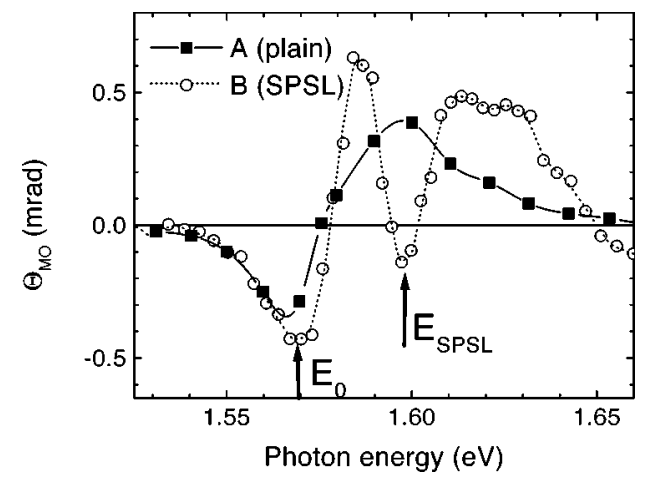

FIG. 4. Photon energy $\left(\omega=\omega_{1}=\omega_{2}\right)$ dependence of the induced Faraday rotation of sample A and B under the conditions of Fig. 3 and at a fixed delay $\Delta t=1 \mathrm{ps} . E_{0}$ and $E_{\mathrm{SPSL}}$ indicate the approximate energies of the lowest optical transition in the QW and SPSL barrier, respectively.

In the analysis of the TIMMS data we concentrate on the $1 F$ signal, which carries most of the information. The pumpinduced Faraday rotation is calculated from the experiment by applying Eq. (14), and is represented in the top panels for a number of photon energies. Sample A shows for $t>1 \mathrm{ps}$ an almost perfect exponential decay with time constant $\tau_{s}$ $\sim 15$ ps. Although this relaxation time only slightly depends on the photon frequency, the amplitude of the induced rotation changes sign while scanning through the band gap. This is shown in more detail in Fig. 4, where $\Theta_{\mathrm{MO}}$ is plotted at fixed delay, $\Delta t=1 \mathrm{ps}$, as function of the photon energy. As expected, the induced signal disappears at energies well below the band gap. The resonant structure around the band gap, as well as the occurrence of the zero crossing will be explained in Sec. V.

The presence of the SPSL in the barriers of sample B significantly affects the spin dynamics and its spectroscopy. The dominant signal has a decay time quite close to that of A: $\tau_{s}=10-12 \mathrm{ps}$. However, for frequencies around the SPSL transition and above, an additional faster dynamical component is found with a spin scattering time of $\tau_{s}=4 \mathrm{ps}$. Also the sign of this second component changes while scanning through its corresponding resonance, as seen by the different curvature of the 1.596 and $1.617 \mathrm{eV}$ curves in Fig. 2, and the resonant structure in Fig. 4.

For comparison, the transient transmission data for the same samples are shown in the bottom panels of Fig. 3 . These were obtained by inserting a polarizer after the PEM, as discussed in Sec. III. This configuration is insensitive to the magnetic or orientational effects, and only resolves the intensity dependent transmission (related to the saturable absorption) due to state filling and possible thermal effects. The observation of a completely contrasting dynamics, viz. a much slower exponential decay ( $\tau \geqslant 200 \mathrm{ps}$ ), clearly demonstrates that the TIMMS and transient transmission experiments probe different dynamics, viz. that of the spins and carriers, respectively. Apart from the slow dynamical component, however, a faster $\Delta T / T$ signal is present in most scans. This carrier relaxation has a time scale which is quite similar to the slower spin process, $\tau_{c} \sim 10-20 \mathrm{ps}$, its amplitude and relaxation rate depending on details of the sample and frequency. At present, we do not fully understand the 
origin of this component. Regarding a possible 4 ps component, present in the spin response of sample $\mathrm{B}$, we can definitely say that there is no sign of this in the carrier response.

\section{DISCUSSION}

Recent work of Buss et al. on a giant photoinduced Faraday rotation deals with heterostructures that are quite similar to ours, viz. ten periods of $47 \AA$ thick CdTe wells, and $\mathrm{Cd}_{1-x} \mathrm{Mn}_{x} \mathrm{Te}(x=0.17)$ barriers. ${ }^{6}$ At zero delay and $T$ $=5 \mathrm{~K}$ a photoinduced Faraday spectrum was found with a maximum rotation of $0.1 \mathrm{rad}$, however using $20 \mathrm{ps}$ pulses with a higher fluence of $10 \mu \mathrm{J} / \mathrm{cm}^{2}$. Thus, their low temperature nonlinear MO efficiency, $\chi_{\mathrm{MO}}=0.1 / 10^{-5}=10^{4} \mathrm{~cm}^{2} / \mathrm{J}$, is two orders of magnitude higher than our room temperature result, $\chi_{\mathrm{MO}}=2 \times 10^{-4} / 10^{-6}=200 \mathrm{~cm}^{2} / \mathrm{J}$. This is well explained by the reported enhancement of the $g$ factor (up to a value of 100) in DMS materials at low $T .{ }^{14}$ A recovery time of $\sim 100 \mathrm{ps}$ was found for the photoinduced Faraday rotation in a magnetic field of $1 \mathrm{~T}$.

Spin scattering in DMS heterostructures at zero field was reported by Baumberg et al., who found $\tau_{s}=6 \mathrm{ps}$ at $T$ $=4 \mathrm{~K}$ in $\mathrm{ZnSe} / \mathrm{ZnMnSe}$ QWs. ${ }^{5}$ In GaAs/AlGaAs nonmagnetic semiconductor QWs, at room temperature an instantaneous spin relaxation for the holes $(<1 \mathrm{ps})$, and a $30 \mathrm{ps}$ electron spin relaxation time was found, ${ }^{16}$ whereas spin relaxation times of $50 \mathrm{ps}$ (holes) and 100-200 ps (electrons) where found at $4 \mathrm{~K} .{ }^{17}$ Thus, we may ascribe the $15 \mathrm{ps}$ spin relaxation component to the electrons, while the instantaneous peak around zero delay (Fig. 2) might be due to the holes. In addition, the appearance of the 4 ps component in sample B could be assigned to electron states in the SPSL, since their larger overlap with the $\mathrm{Cd}_{1-x} \mathrm{Mn}_{x} \mathrm{Te}$ magnetic barriers is expected to enhance the spin-flip rate. Although certainly more detailed experiments are required for a definite interpretation, we can certainly conclude that the spin relaxation rates are in reasonable agreement with former literature.

In order to understand the dispersion of the TIMMS signal (Fig. 4) it is important to realize that: (i) in our experiments $\omega_{1}=\omega_{2}$, and (ii) the $V_{1 F}$ signal is proportional to the real part of the complex magneto-optical rotation [Eq. (14)]. In transmission $V_{1 F}$ is proportional to the real part of $\epsilon_{x y}$ (assuming the diagonal element of $\epsilon$ to be real, which approximately holds just above the fundamental band gap).

Right after arrival of the pump pulse, the oscillator strength at $\omega$ is depleted. This causes a peak in the imaginary (dichroic) part of $\epsilon_{x y}\left(\epsilon_{x y}^{\prime \prime}\right)$ at the pump and probe frequency. However, the real part of $\epsilon_{x y}\left(\epsilon_{x y}^{\prime}\right)$, which is related to $\epsilon_{x y}^{\prime \prime}$ by a Kramers-Kronig transformation, is still zero. Only after the first relaxation of excitations toward lower energies has occurred, does $\epsilon_{x y}^{\prime}$ start to grow. Excitations relax towards a quasistable equilibrium at the bottom of the band, with a total width of approximately $k_{B} T \sim 25 \mathrm{meV}$. At this time the new population has become almost independent of the pump frequency. The $\epsilon_{x y}^{\prime \prime}$ profile closely resembles the new joined density of states. The corresponding (Kramers-Kronig resolved) $\epsilon_{x y}^{\prime}$ is negative below and positive above $E_{0}$. The TIMMS spectrum is given by the product of the absorption of the pump beam, a broadened step function around $E_{0}$, and $\epsilon_{x y}^{\prime}(\omega)$. Thus, the difference in absorption below and above $E_{0}$ explains the difference in amplitude of the low energy negative and larger energy positive peaks in the TIMMS signal.

After a spin-lattice relaxation time of $\tau \sim 15 \mathrm{ps}$ the inequivalent spin population starts to balance, and the magnetic signal (exponentially) dies out. Still, excitations will survive at the bottom of the band for a time scale of the $e-h$ recombination time which we found to be $\tau_{c}>200 \mathrm{ps}$ in the $\Delta T / T$ experiment.

This satisfactorily explains the TIMMS spectrum of sample A in Fig. 4. The widths of the positive feature approximately correspond to $k_{B} T \sim 25 \mathrm{meV}$ at room temperature. Also note that the temporal width of our laser pulses (70 fs) yields an additional line broadening. Furthermore we note that even at room temperature exciton effects are still not fully negligible in QW structures, which may result in an additional enhancement and sharpening of the TIMMS signal around the band gap. A more detailed interpretation of the spectroscopy and its ultrafast evolution will be reported in a forthcoming paper. ${ }^{18}$

The doubly resonant structure found for sample B is explained by the presence of a similar resonance at the SPSL gap. The fact that a significant 4 ps component, ascribed to the electron spin relaxation in the SPSL, is found in the spin dynamics, but not in the carrier dynamics, implies that the tunnel rate of electrons to the QW is lower than the spin-flip rate in the SPSL.

At negative delay we measured no detectable Faraday rotation, indicating the absence of slow ( $>$ ns) dynamics. This is in contrast with similar experiments on $\mathrm{Zn}_{0.76} \mathrm{Mn}_{0.24} \mathrm{Se} / \mathrm{Zn}_{0.77} \mathrm{Cd}_{0.23} \mathrm{Se}$ quantum structures at low temperature, in which a slow dynamics at $\mu$ s has been reported. ${ }^{5}$ Probably, magnetic imprinting of the magnetic ions, which was thought to be the origin of this long-lived signal, is absent (or at least much faster) at room temperature, far above any spin-glass behavior.

In order to study this magnetic imprinting effect, as well as the temperature dependence of the spin dynamics, it might be of particular interest to perform temperature dependent TIMMS. The possibility of performing a phase sensitive analysis [Eq. (18)] provided by the modulation scheme, will offer a flexible scheme to study ultrafast and long-lived signals in the same experiment. Moreover, an extreme enhancement of the signals, and thus of the relative sensitivity, is expected at low temperatures due to the enhancement of the MO response. ${ }^{14}$

\section{CONCLUSIONS}

Summarizing, we introduced TIMMS as a new technique for studying ultrafast spin dynamics. We demonstrated a high magneto-optical sensitivity of $\sim 10^{-7} \mathrm{rad}$, achieved by using a modulation scheme, an almost crossed polarizer/ analyzer combination, and a state-of-the-art solid state pump laser. A quantitative analysis of the signals in this configuration [Eqs. (14) and (15)] revealed the dominant character of the $1 F$ signal, which was experimentally confirmed. First 
experiments on $\mathrm{CdTe} / \mathrm{CdMnTe} \mathrm{QWs}$ displayed a spin-lattice relaxation time of approximately $15 \mathrm{ps}$, almost independent of excitation/probe frequency. An additional, faster component is found for samples with a SPSL barrier structure, possibly because of the enhanced spin scattering induced by the larger overlap of the barrier wave functions with the magnetic ions. Many details of the spin and carrier dynamics are still unclear, however, and additional experiments will be needed to achieve a more complete understanding of the dynamics, both at fast (10 ps) and ultrafast (subpicosecond) time scales. Interesting new results may be expected from two-color experiments $\left(\omega_{1} \neq \omega_{2}\right)$, tuning of the QW parameters (such as the composition $x$, and the $\mathrm{QW}$ and barrier width) and experiments at low temperature. Such extensions are a matter of current investigations.

Circularly pumping is usually less efficient in metals due to the quenching of the orbital angular momentum by the crystal fields. We conjecture that due to its extreme sensitivity TIMMS might serve as an interesting complementary probe of the spin dynamics of metallic systems as well, touching the complicated but fascinating field of femtosecond spin dynamics in itinerant ferromagnets.

\section{ACKNOWLEDGMENTS}

The authors thank H. J. M. Swagten for carefully reading the manuscript. This work was supported by the Netherlands Foundation for Fundamental Research on Matter (FOM), and by the Polish State Committee for Scientific Research (Grant No. 2P03B119 14).
${ }^{1}$ E. Beaurepaire, J.-C. Merle, A. Daunois, and J.-Y. Bigot, Phys. Rev. Lett. 76, 4250 (1996).

${ }^{2}$ J. Hohlfeld, E. Matthias, R. Knorren, and K. H. Bennemann, Phys. Rev. Lett. 78, 4861 (1997).

${ }^{3}$ A. Y. Elezzabi, M. R. Freeman, and M. Johnson, Phys. Rev. Lett. 77, 3220 (1996).

${ }^{4}$ W. K. Hiebert, A. Stankiewicz, and M. R. Freeman, Phys. Rev. Lett. 79, 1134 (1997)

${ }^{5}$ J. J. Baumberg, S. A. Crooker, D. D. Awschalom, N. Samarth, H. Luo, and J. Furdyna, Phys. Rev. B 50, 7689 (1994).

${ }^{6}$ C. Buss, R. Pankoke, P. Leisching, J. Cibert, R. Frey, and C. Flytzanis, Phys. Rev. Lett. 78, 4123 (1997).

${ }^{7}$ G. Ju, A. Vertikov, A. V. Nurmikko, C. Canady, G. Xiao, R. F. C. Farrow, and A. Cebollada, Phys. Rev. B 57, R700 (1998).

${ }^{8}$ M. Aeschlimann, M. Bauer, S. Pawlik, W. Weber, R. Burgermeister, D. Oberli, and H. C. Siegmann, Phys. Rev. Lett. 79, 5158 (1997).

${ }^{9}$ A. Scholl, L. Baumgarten, R. Jacquemin, and W. Eberhardt, Phys. Rev. Lett. 79, 5146 (1997).

${ }^{10}$ D. D. Awschalom, N. R. Freeman, N. Samarath, H. Luo, and J. K. Furdyna, Phys. Rev. Lett. 66, 1212 (1991).

${ }^{11}$ M. R. Freeman, D. D. Awschalom, J. M. Hong, and L. L. Chang, Phys. Rev. Lett. 64, 2430 (1990).

${ }^{12}$ J. A. Gaj, in Semiconductors and Semimetals, edited by R. K. Willardson, A. C. Beer, J. K. Furdyna, and J. Kossut (Academic, Boston, 1988), Vol. 25.

${ }^{13}$ W. J. M. de Jonge and H. J. M. Swagten, J. Magn. Magn. Mater. 100, 322 (1991).

${ }^{14}$ J. A. Gaj, R. R. Galazka, and M. Nawrocki, Solid State Commun. 25, 193 (1978).

${ }^{15}$ S. Koshihara, A. Oiwa, M. Hirasawa, S. Katsumoto, Y. Iye, C. Urano, H. Takagi, and H. Munekata, Phys. Rev. Lett. 78, 4617 (1997).

${ }^{16}$ A. Tackeuchi, S. Muto, T. Inata, and T. Fujii, Appl. Phys. Lett. 56, 2213 (1990).

${ }^{17}$ S. Bar-Ad and I. Bar-Joseph, Phys. Rev. Lett. 68, 349 (1992).

${ }^{18}$ B. Koopmans and W. J. M. de Jonge, Appl. Phys. B 68, 525 (1999). 